\title{
Estructura del bosque altoandino y páramo en el Macizo de Bijagual, Boyacá, Colombia
}

\section{Pablo Andrés Gil-Leguizamón ${ }^{1 *}$, María Eugenia Morales-Puentes ${ }^{2} \&$ Jorge Jácome $^{3}$}

1. Grupo Sistemática Biológica-SisBio, Herbario UPTC, ViE-DIN, Escuela de Ingeniería Ambiental, Facultad de Ingeniería, Universidad Pedagógica y Tecnológica de Colombia, Av. Central del Norte 39-115, Campus Universitario, Edificio Centro de Laboratorios, Tunja-Boyacá, Colombia; pablo.gil@uptc.edu.co

2. Grupo Sistemática Biológica-SisBio, Herbario UPTC, ViE-DIN, Escuela de Biología, Facultad de Ciencias, Universidad Pedagógica y Tecnológica de Colombia, Av. Central del Norte 39-115, Campus Universitario, Edificio Centro de Laboratorios, Tunja-Boyacá, Colombia; maria.morales@uptc.edu.co

3. Unidad de Ecología y Sistemática (UNESIS), Departamento de Biología, Facultad de Ciencias, Pontificia Universidad Javeriana, Bogotá, Colombia; jacomej@javeriana.edu.co

* Correspondencia

Recibido 22-IV-2020. Corregido 13-V-2020. Aceptado 15-V-2020.

\begin{abstract}
Structure of the high Andean forest and paramo in the Bijagual Massif, Boyacá, Colombia. Introduction: Colombia has increased research due to the necessity of knowing the country's biodiversity and ecosystems, as well as to establish priorities for their protection throughout the national territory. The knowledge of the Boyacá ecosystems and the high Andes are not an exception. Objective: this work provides information on diversity and structure of the Bijagual Massif, a high mountain area defined as a biological corridor, with paramo (2 900 - 3460 masl) and high Andean forest (2 682 -3 268 masl) ecosystems. Methods: Sampling consisted of 27 random transects in the high Andean forest $(100 \times 2.5 \mathrm{~m})$ and 13 in the paramo $(10 \times 5 \mathrm{~m})$. We recorded abundance data, growth form, height, coverage, DAP and basal area. We calculated diversity analyses (H', D and Jaccard), of vertical and horizontal structure. Results: Estimated species richness of 429 species in 86 families. Similarity does not exceed $46 \%$ between sampling units, data that supports the heterogeneity of the Bijagual Massif. We recorded 10777 individuals in the forest. The most abundant and richest growth forms were herbs (2 595/104) and trees (2 189/67), while lower in abundance and richness were vines (466/29). In the paramo 3337 individuals, with a dominance of herbs (in frailejonal, shrubs and pajonal), rosettes (in frailejonal and pajonal) and shrubs. Species with ecological representativeness are: Clusia multiflora, C. elliptica, C. alata, Weinmannia rollottii, Brunellia comocladifolia and Viburnum triphyllum. Conclusion: These results confirm the uniqueness of these high mountain ecosystems and the need to maintain the heterogeneity before the occurence of soil transformation processes. The last, is supported by the high species turnover among sampling units, the dominance per physiognomic type, and the ecological significance of arboreal and shrub species in the forest and those that reoccur in the paramo. Here we define Bijagual, as a biological corridor with great biodiversity.
\end{abstract}

Key words: high-Andean forest; paramo; diversity; forms of growth; vegetation.

Gil-Leguizamón, P.A., Morales-Puentes, M.E., \& Jácome, J. (2020). Estructura del bosque altoandino y páramo en el Macizo de Bijagual, Boyacá, Colombia. Revista de Biología Tropical, 68(3), 765-776.

La vegetación es el arreglo integral de factores bióticos y abióticos; las especies de una comunidad están en consonancia con la variación geográfica y el medio ecológico en el que se desarrollan, factores que determinan la variación espacial y fisionómica de la vegetación (Etter \& van Wynngaarden, 2000; Cleef, 2013).

Conocer la expresión fisionómica y estructural de la vegetación implica identificar patrones de distribución y dominancia, abundancia, frecuencia, arreglo ecológico horizontal 
y vertical de las especies de la comunidad (Gentry, 1988; Rangel \& Velásquez, 1997).

La caracterización estructural permite definir el estado de conservación de la vegetación, siendo significativa en la región Andina donde, articulado al desarrollo cultural y económico, evidencia la transformación del paisaje natural en agrosistemas extensivos e intensivos (Etter \& van Wynngaarden, 2000; Armenteras et al., 2011; Armenteras, Cabrera, Rodríguez, \& Retana, 2013; Gil \& Morales, 2015).

El Macizo de Bijagual (Boyacá), es un escenario que cobra interés especial por su potencial en biodiversidad, a partir de la caracterización de vegetación, siendo un aporte de nuevo y relevante conocimiento de la estructura de los ecosistemas de la alta montaña, aplicable a estudios de ecología funcional y restauración de áreas degradadas (Gil, 2016).

Como información conjunta al estudio florístico realizado por Carrillo, Morales-Puentes y Gil-Leguizamón (2017), este trabajo proporciona información sobre la diversidad y estructura de las formaciones vegetales del bosque altoandino y páramo en Bijagual.

\section{MATERIALES Y MÉTODOS}

Área de estudio: el Macizo de Bijagual es un corredor biológico de alta montaña ubicado en la zona centro-sur del departamento de Boyacá, Colombia (526'10.28' - 5¹5'40.21" N \& 7313'5.20” - 73²1'58.19” W) (MoralesPuentes, Gil-Leguizamón, Simbaqueba-Gutiérrez, \& Carrillo-Fajardo, 2012). La extensión es de 8604 ha; con páramo entre 2990-3460 m de altitud y bosque altoandino entre 2682-3268 $\mathrm{m}$; limita con el complejo de páramos de Tota y la Reserva Natural Mamapacha (Gil, 2016). Precipitación entre 1000-2500 mm/anual y temperatura entre $13-17{ }^{\circ} \mathrm{C}$ (Carrillo et al., 2017; Gil \& Morales, 2017).

Muestreo: se realizaron 27 transectos aleatorios en bosque altoadino $(100 \times 2.5 \mathrm{~m}=$ $0.68 \mathrm{ha})$ y 13 en páramo $(10 \times 5 \mathrm{~m}=0.07 \mathrm{ha})$ en tipos fisionómicos de frailejonal, arbustal y pajonal, según Rangel y Velásquez (1997).
Para cada individuo se registró la abundancia (clonales: porcentaje de cobertura), forma de crecimiento (árbol, arbusto, hierba, bejuco, epífita, hemiepífita, helecho arborescente, parásita, rasante, roseta y graminoide, según Font, 2001), altura $(\mathrm{m})$, cobertura $\left(\mathrm{m}^{2}\right)$, diámetro a la altura del pecho (DAP) para árboles y área basal $(\mathrm{AB})$ para arbustos.

Trabajo de laboratorio: el material botánico se procesó en el Herbario UPTC, se determinó con literatura especializada como Gentry (1993), Flora de Colombia, Flora Neotrópica, Flora de Costa Rica, Flora de Panamá, entre otras, y páginas web (www.tropicos.org, www.botanicus.orgs y www.jstor.org). Cada muestra fue corroborada con los Herbarios, UPTC, COL y HECASA y especialistas. Los ejemplares se depositaron en UPTC, bajo la numeración de Gil-Leguizamón P.A.

\section{Tratamiento de datos}

Diversidad: se calcularon los estadísticos no paramétricos Chao-1 y ACE que cuantificaron la representatividad del muestreo, a partir de datos de riqueza y abundancia (software EstimateS-6.0). Para cada unidad de muestreo se estimaron los índices de Shannon (H') y Simpson (D) como indicadores de probabilidad de diversidad-dominancia. Se realizó un análisis de composición de especies, mediante aglomeración, con el coeficiente de similitud de Jaccard con el algoritmo de grupos pareados (Hammer, Harper, \& Ryan, 2008) con el software PAST (PAleontological STatistics, versión 3.22).

Estructura vertical: la fisionomía del bosque altoandino y páramo fue descrita de acuerdo a formas de crecimiento, abundancia y riqueza de especies; a partir, de intervalos de clase por altura $(\mathrm{m})$, cobertura $\left(\mathrm{m}^{2}\right)$ y DAP (m) (Rangel \& Velásquez, 1997; basado en la regla de Sturges) se obtuvo la distribución de individuos y su asociación con las formas de crecimiento. Horizontal: para identificar las especies arbóreas y arbustivas dominantes y 
frecuentes en el bosque altoandino y en áreas de arbustos en páramo, se calculó el Índice de Predominio Fisionómico (IPF) y de Valor de Importancia (IVI), a partir de la abundancia, cobertura y área basal (Rangel \& Velásquez, 1997); para frailejonal y pajonal, se estimó el índice de valor de importancia relativa (IVR) (Álvarez-Lopeztello, Rivas-Manzano, Aguilera-Gómez, \& González-Ledesma, 2016).

\section{RESULTADOS}

Diversidad: se registraron 429 especies en 86 familias (396 especies en bosque altoandino y 111 en páramo: 78 compartidas); para el bosque altoandino se evidenció una representatividad de muestreo del $91 \%$ con el estimador Chao-1 y $93 \%$ con ACE; en los dos casos, la riqueza esperada es de 414 y 407 especies respecto a las 396 observadas. En páramo, la representatividad fue del $93 \%$ (Chao-1) y $95 \%$ (ACE), con una riqueza esperada de 120 y 118 especies, comparado con las 111 especies observadas.

Para el bosque altoandino, el índice de Shannon determinó una probabilidad de diversidad de $3.3048( \pm 0.2392)$ para los 27 transectos, lo que puede considerarse como representativo; los valores de dominancia de Simpson son inversos a la diversidad (0.0591 \pm 0.0192 ). En páramo es similar, aunque con valores inferiores, la diversidad media de Shannon fue de $2.3932( \pm 0.3101)$, y la dominancia inversa para los 13 transectos $(0.1234 \pm 0.0321)$.

Entre unidades de bosque la similitud no superó el $46 \%$ (Fig. 1A). Comunes en estos transectos son: Bomarea setacea (Ruiz \& Pav.) Herb., Brunellia comocladifolia Bonpl., Clusia alata Planch. \& Triana, Clusia elliptica Kunth, Drimys granadensis L. f., Greigia stenolepis L.B. Sm., Hedyosmum colombianum Cuatrec., Macleania rupestris (Kunth) A.C. Sm., Piper irazuanum C. DC. y Weinmannia rollottii Killip. En páramo, la similitud es inferior al $38 \%$, el pajonal es similar en $8 \%$ y solo el transecto 12 de arbustal es disímil (Fig. 1B), por la exclusividad de Diplostephium alveolatum Cuatrec., Bomarea hirsuta (Kunth) Herb., Fuchsia canescens Benth., Pentacalia tolimensis (Sch. Bip. ex Wedd.) Cuatrec. y Symplocos cundinamarcensis B. Ståhl. Solo Diplostephium phylicoides (Kunth) Wedd. fue registrada en los tres tipos fisionómicos.
A

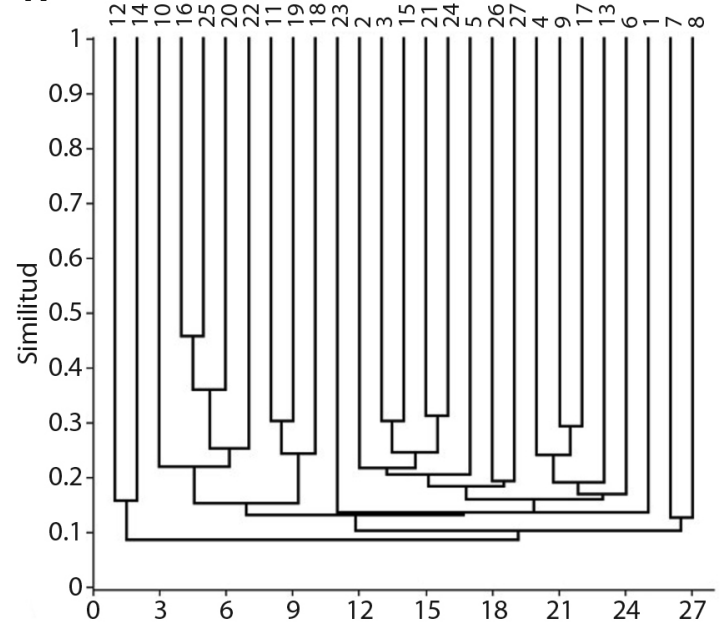

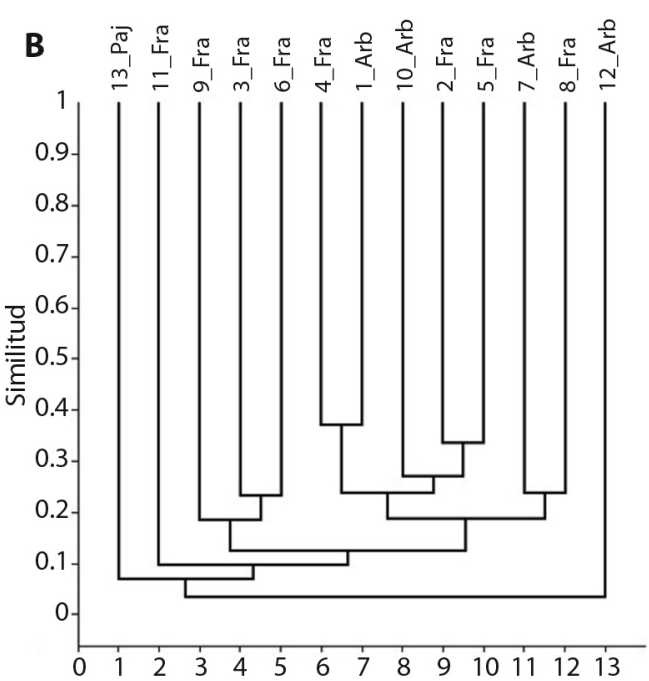

Fig. 1. Dendrogramas basados en el coeficiente de similitud de Jaccard y algoritmo de grupos pareados para cada unidad de muestreo. A. Bosque altoandino B. Páramo (Fra: Frailejonal; Arb: Arbustal; Paj: Pajonal).

Fig. 1. Dendrograms based on Jaccard's similarity coefficient and algorithm matched group for each sampling unit. A. HighAndean forest B. Paramo (Fra: Frailejonal; Arb: Arbustal; Paj: Pajonal). 


\section{Estructura vertical}

Formas de crecimiento en bosque altoandino: se registraron 10777 individuos. Fisionómicamente, el bosque tiene dominio de individuos arbóreos hasta de $18 \mathrm{~m}$ de altura $(\bar{x} 8.45 \mathrm{~m}, \pm 3.7 \mathrm{~m})$, en zonas planas a moderadamente inclinadas $\left(5-20^{\circ}\right)$. Se identificaron 11 formas, siendo dominantes, árboles con el $20 \%$ de la abundancia (2 189 individuos) y el $17 \%$ de la riqueza (67 especies). En el sotobosque dominan arbustos (16\%, 1729 individuos; $15 \%, 59$ especies), epífitas (19\%, 2057; $25 \%$, 99) y herbáceas $(24 \%, 2595 ; 26 \%, 104)$, éstas últimas son las más abundantes y ricas. Otras formas fueron: bejucos (466 individuos / 29 especies), hemiepífitas (305/5), helechos arborescentes (99/8), rasantes (81/6), rosetas (1211/12), graminoides (tres especies clonales) y parásitas (45/4) (Tabla 1).

Formas de crecimiento en páramo: Se registraron 3337 individuos, el crecimiento se describe por tipos de vegetación, así (Tabla 1): Frailejonal: conformado por individuos en roseta y arbustos $\leq 3.5 \mathrm{~m}$ de altura $(\overline{\mathrm{x}} 0.96 \mathrm{~m}, \pm$ $0.83 \mathrm{~m}$ ), distribuidos en zonas planas o moderadamente inclinadas. Se registraron 2342 individuos, en 73 especies y nueve formas; las rosetas fueron dominantes con el $36 \%$ de la abundancia (876) y $18 \%$ de la riqueza (13), así como las hierbas $(44 \%, 1052 ; 34 \%, 25)$ y arbustos $(14 \%, 346 ; 26 \%, 19)$. Otras formas fueron: plántulas de árboles $(16 / 5), \leq 90 \mathrm{~cm}$ de altura (Geissanthus andinus Mez, Oreopanax integrifolium Cuatrec., Pentacalia trichopus (Benth.) Cuatrec., W. fagaroides y W. rollottii), rasantes (33/4), gramíneas (3 spp.), bejucos $(10 / 1)$, epífitas $(8 / 2)$ y hemiepífita $(1 / 1)$.

Arbustal: con alturas $\leq 3.1 \mathrm{~m}(\overline{\mathrm{x}} 1.3 \mathrm{~m}, \pm$ $1 \mathrm{~m})$ sobre pendientes leves $\left(<10^{\circ}\right)$, moderadas $\left(<20^{\circ}\right)$ a pronunciadas $\left(20-35^{\circ}\right)$. Se registraron 782 individuos, en 66 especies y ocho formas; los arbustos fueron dominantes con el $33 \%$ de la abundancia (272) y el $27 \%$ de la riqueza (18 spp.), en su orden, las hierbas $(27 \%, 221 ; 35 \%, 23)$, rosetas $(18 \%, 151 ; 9$ $\%, 6)$ y plántulas de árboles $(10 \%, 82 ; 12 \%$, 8), como $W$. fagaroides, G. andinus, W. rollottii y $W$. tomentosa. De menor abundancia y riqueza fueron: los bejucos (33/6), epífitas (11/2), rasantes (12/2) y gramíneas como $C$. nitida.

Pajonal: hasta $1.15 \mathrm{~m}(\overline{\mathrm{x}} 0.56 \mathrm{~m}, \pm 0.29$ $\mathrm{m})$. Se hallaron 213 individuos en 12 especies y cinco formas; $C$. effusa fue dominante con el $70 \%$ de la cobertura en el muestreo, otras dominantes fueron las hierbas $(64 \%, 172$; $67 \%, 8)$ y rosetas $(14 \%, 37 ; 8 \%, 1)$, en su

TABLA 1

Especies más abundantes por formas de crecimiento en Bijagual

TABLE 1

Most abundant species by growth forms in Bijagual

\begin{tabular}{lcc}
\multicolumn{1}{c}{ Especie } & Formas de crecimiento & Abundancia o \% cobertura \\
Bosque altoandino & & 192 \\
Weinmannia rollottii Killip & Árbol & 136 \\
Clusia elliptica Kunth & Árbol & 118 \\
Drimys granadensis L. f. & Árbol & 98 \\
Brunellia comocladifolia Bonpl. & Árbol & 178 \\
Hedyosmum colombianum Cuatrec. & Arbusto & 108 \\
Disterigma alaternoides (Kunth) Nied. & Arbusto & 106 \\
Macleania rupestris (Kunth) A.C. Sm. & Arbusto & 195 \\
Melpomene moniliformis (Lag. ex Sw.) A.R. Sm. \& R.C. Moran & Epífita & 179 \\
Hymenophyllum polyanthos (Sw.) Sw. & Epífita & 115 \\
Fernandezia lanceolata (L.O. Willams) Garay \& Dunst. & Epífita & 359 \\
Neurolepis aperta (Munro) Pilg. & Hierba & \\
\hline
\end{tabular}


TABLA 1 (Continuación) / TABLE 1 (Continued)

\begin{tabular}{|c|c|c|}
\hline Especie & Formas de crecimiento & Abundancia o \% cobertura \\
\hline Piper irazuanum C. DC. & Hierba & 102 \\
\hline Begonia urticae L. f. & Hierba & 54 \\
\hline Mikania aristei B.L. Rob. & Bejuco & 45 \\
\hline Bomarea setacea (Ruiz \& Pav.) Herb. & Bejuco & 59 \\
\hline Anthurium oxybelium Shott & Hemiepífita & 67 \\
\hline Cyathea meridensis H. Karst. & Helecho arborescente & 29 \\
\hline Cyathea caracasana (Klotzsch) Domin & Helecho arborescente & 22 \\
\hline Nertera granadensis (Mutis ex L. f.) Druce & Rasante & 23 \\
\hline Blechnum auratum (Fée) R. M. Tryon \& Stolze & Roseta & 218 \\
\hline Blechnum schomburgkii (Klotzsch) C. Chr. & Roseta & 174 \\
\hline Greigia stenolepis L.B. Sm. & Roseta & 106 \\
\hline Cortaderia nitida (Kunth) Pilg. & Graminoide & $3 \%$ \\
\hline Dendrophthora lindeniana Tiegh. & Parásita & 28 \\
\hline \multicolumn{3}{|l|}{ Páramo-Frailejonal } \\
\hline Espeletia murilloi Cuatrec. & Roseta & 337 \\
\hline Paepalanthus columbiensis Ruhland & Roseta & 177 \\
\hline Puya goudotiana $\mathrm{Mez}$ & Roseta & 109 \\
\hline Chusquea tessellata Munro & Hierba & 183 \\
\hline Rhynchospora ruiziana Boeckeler & Hierba & 208 \\
\hline Calamagrostis effusa (Kunth) Steud. & Graminoide & $7 \%$ \\
\hline Hypericum lycopodioides Triana \& Planch. & Arbusto & 34 \\
\hline Diplostephium phylicoides (Kunth) Wedd. & Arbusto & 18 \\
\hline Gaultheria erecta Vent. & Rasante & 19 \\
\hline Bomarea setacea (Ruiz \& Pav.) Herb. & Bejuco & 10 \\
\hline Epidendrum decurviflorum Schltr. & Epífita & 1 \\
\hline Anthurium oxybelium Shott & Hemiepífita & 1 \\
\hline \multicolumn{3}{|l|}{ Páramo-Arbustal } \\
\hline Monochaetum myrtoideum Naudin & Arbusto & 31 \\
\hline Macleania rupestris (Kunth) A.C. Sm. & Arbusto & 22 \\
\hline Hypericum lycopodioides Triana \& Planch. & Arbusto & 21 \\
\hline Ageratina tinifolia (Kunth) R.M. King \& H. Rob. & Arbusto & 18 \\
\hline Carex confertospicata Boeckeler & Hierba & 36 \\
\hline Chusquea tessellata Munro & Hierba & 26 \\
\hline Disterigma empetrifolium (Kunth) Drude & Hierba & 20 \\
\hline Blechnum auratum (Fée) R. M. Tryon \& Stolze & Roseta & 107 \\
\hline Bomarea setacea (Ruiz \& Pav.) Herb. & Bejuco & 16 \\
\hline Epidendrum frutex $\mathrm{Rchb} . \mathrm{F}$. & Epífita & 1 \\
\hline Galium hypocarpium (L.) Endl. ex Griseb. & Rasante & 6 \\
\hline Cortaderia nitida (Kunth) Pilg. & Graminoide & $11 \%$ \\
\hline \multicolumn{3}{|l|}{ Páramo-Pajonal } \\
\hline Calamagrostis effusa (Kunth) Steud. & Graminoide & $70 \%$ \\
\hline Rhynchospora ruiziana Boeckeler & Graminoide & 43 \\
\hline Neurolepis acuminatissima (Munro) Pilg. & Hierba & 43 \\
\hline Bartsia santolinifolia (Kunth) Benth. & Hierba & 21 \\
\hline Paepalanthus pilosus Kunth & Roseta & 37 \\
\hline Diplostephium phylicoides (Kunth) Wedd. & Arbusto & 1 \\
\hline Jamesonia rotundifolia feé & Rasante & 3 \\
\hline
\end{tabular}


orden rasantes (3 spp.) y un individuo arbustivo de $D$. phylicoides.

\section{Intervalos de clase en bosque altoandino}

Altura (intervalos: 16; amplitud de clase: $1.14 \mathrm{~m}$ ): entre 0.01 y $4.6 \mathrm{~m}$ se agrupa el $86 \%$ de la abundancia (8 424 individuos). En este rango, las hierbas se distribuyen entre $0.01 \mathrm{y}$ $2.3 \mathrm{~m}$ (6 809 individuos, estrato inferior - intervalos 1 y 2) y los arbustos entre 2.31 y $4.6 \mathrm{~m}$ (1 615, estrato intermedio - intervalos 3 y 4). Alturas superiores a 4.61-18.5 m (intervalos 5 a 16), agrupan el $14 \%$ de la abundancia (1 338, estrato superior) con especies de sotobosque y dosel. Árboles de mayor altura son: Brunellia colombiana Cuatrec., Brunellia propinqua Kunth, Clusia multiflora Kunth, Gaiadendron punctatum (Ruíz \& Pav.) G. Don., Hieronyma rufa P. Franco, Ilex obtusata Triana \& Planch., Morella funckii (Chev.) Parra-Os., Prunus opaca (Benth.) Walp., Pleurothyrium velutinum Meisn., Ternstroemia camelliifolia Linden \& Planch., W. balbisiana y W. rollottii.

Cobertura (intervalos: 16; amplitud de clase: $15.3 \mathrm{~m}^{2}$ ): el $94 \%$ de los individuos con coberturas hasta $15.3 \mathrm{~m}^{2}$ (9 245 - intervalo 1); el $5 \%$ (453) entre 15.3 y $45.9 \mathrm{~m}^{2}$ (intervalos 2 y 3 ) y el $2 \%$ (166) entre 46 y $152 \mathrm{~m}^{2}$ (intervalos 4 a 10); solo $C$. alata con un área de copa, hasta $240 \mathrm{~m}^{2}$ (intervalos 11 a 16). Otras de amplia cobertura son: B. colombiana, $B$. comocladifolia, B. propinqua, C. multiflora, I. obtusata, M. funckii y P. opaca.

Diámetro (DAP; intervalos: 14; amplitud de clase: $0.64 \mathrm{~m}$ ): el $87 \%$ (2 543 individuos) presentó diámetros de tronco entre 0.01 y 0.177 m (intervalos 1 a 3), incluidas plántulas de $C$. alata, D. granadensis, $W$. rollottii, $B$. colombiana, Freziera bonplandiana Tul., Miconia cundinamarcensis Wurdack, M. funckii y Viburnum triphyllum Benth. El $10 \%$ (294 individuos) con diámetros entre 0.178 y $0.345 \mathrm{~m}$ (intervalos 4 a 6) y $3 \%$ (87) con diámetros superiores a $0.346 \mathrm{~m}$ hasta $0.774 \mathrm{~m}$ (intervalos $7 \mathrm{a} 14$ ); de fustes amplios son: $C$. elliptica, $C$. alata, $C$. multiflora, B. colombiana, B. comocladifolia, $W$. balbisiana y $W$. rollottii.

\section{Intervalos de clase en páramo}

Altura (intervalos: 14; amplitud de clase: $0.248 \mathrm{~m}$ ): el $78 \%$ de los individuos (entre hierbas, gramíneas, rosetas y arbustos) con porte entre 0.02 y $1.029 \mathrm{~m}$ (2 685 individuos - intervalos 1 a 4); abundantes en este rango son: $E$. murilloi (frailejonal), $R$. ruiziana, C. tessellata, C. effusa, P. columbiensis (frailejonal y pajonal), $P$. goudotiana, B. auratum, D. phylicoides, R. macrochaeta, V. floribundum, P. prostrata e Hypericum laricifolium Juss. (arbustal). El 21 $\%$ (709) entre 1.03 y $2.5 \mathrm{~m}$ (intervalos 5 a 10) y $1 \%$ (50) entre 2.6 y $3.5 \mathrm{~m}$ (intervalos 11 a 14 ), con: E. murilloi (frailejonal), Diplostephium floribundum (Benth.) Wedd., D. granadensis, Escallonia myrtilloides L. f., O. integrifolium, P. trichopus, S. cundinamarcensis, Ageratina tinifolia (Kunth) R.M. King \& H. Rob., B. glutinosa, G. andinus, M. rupestris, T. grossa, $W$. tomentosa y $W$. rollottii (arbustal).

Cobertura (intervalos: 15; amplitud de clase: $0.76 \mathrm{~m}^{2}$ ): individuos con valores hasta de $0.76 \mathrm{~m}^{2}$ son abundantes $(88 \%, 3067$ intervalo 1), con R. macrochaeta, E. murilloi, C. tessellata, $P$. columbiensis y $D$. phylicoides. Solo el $2 \%$ de los individuos (75) tienen coberturas entre $0.73-11 \mathrm{~m}^{2}$ (intervalos 2 a 15), con A. tinifolia, $H$. lycopodioides, $M$. rupestris, $P$. tolimensis, $P$. trichopus, $S$. cundinamarcensis, $W$. tomentosa, $G$. andinus, B. resinosa, D. alveolatum, $D$. alaternoides, $D$. granadensis y E. myrtilloides.

Diámetro (DAP; 10 intervalos; amplitud de clase: $0.022 \mathrm{~m}$ ): el $98 \%$ de los individuos (366) tienen DAP entre 0.01 y $0.078 \mathrm{~m}$ (intervalos 1 a 3), con $H$. lycopodioides, $W$. fagaroides, M. myrtoideum, G. anastomosans, D. floribundum y T. grossa. El $2 \%$ (9) entre 0.079 y 0.239 m (intervalos 4 a 10) con $D$. floribundum y D. granadensis.

\section{Estructura horizontal}

Índice de Predominio Fisionómico (IPF) en bosque altoandino: del conjunto de especies arbóreas y arbustivas, las dominantes son: C. multiflora, C. elliptica, C. alata y $W$. rollottii (Tabla 2). La cobertura es el parámetro 
TABLA 2

Especies con mayor representatividad ecológica (IPF, IVI e IVR) en Bijagual

TABLE 2

Species with greater ecological representativeness (IPF, IVI and IVR) in Bijagual

\begin{tabular}{|c|c|c|c|c|c|c|}
\hline \multicolumn{7}{|c|}{ Bosque Altoandino } \\
\hline Especie & Presencia & $\mathrm{AbR}$ & \multicolumn{2}{|r|}{$\mathrm{CoR}$} & DeR & IPF \\
\hline Clusia multiflora (A) & $7 / 27$ & 78.1 & \multicolumn{2}{|r|}{73.5} & 2 & 153.5 \\
\hline Clusia elliptica (A) & $23 / 27$ & 99.5 & \multicolumn{2}{|r|}{20.9} & 2.7 & 123.1 \\
\hline Clusia alata (A) & $15 / 27$ & 27.8 & \multicolumn{2}{|r|}{68} & 22.2 & 118 \\
\hline Weinmannia rollottii (A) & $18 / 27$ & 73.1 & \multicolumn{2}{|r|}{33.1} & 4.3 & 110.5 \\
\hline Brunellia comocladifolia (A) & $16 / 27$ & 36.5 & \multicolumn{2}{|r|}{70.1} & 2 & 108.6 \\
\hline Viburnum triphyllum (A) & $8 / 27$ & 42.2 & \multicolumn{2}{|r|}{34.5} & 26.5 & 103.2 \\
\hline Macleania rupestris (Ar) & $19 / 27$ & 2.2 & \multicolumn{2}{|r|}{93.7} & 4.7 & 100.6 \\
\hline Escallonia myrtilloides (A) & $5 / 27$ & 1.1 & \multicolumn{2}{|r|}{71} & 16.3 & 88.3 \\
\hline Especie & Presencia & DeR & \multicolumn{2}{|r|}{ DoR } & FrR & IVI \\
\hline Clusia elliptica (A) & $23 / 27$ & 2.7 & \multicolumn{2}{|r|}{99.5} & 4.9 & 107.1 \\
\hline Viburnum triphyllum (A) & $8 / 27$ & 26.5 & \multicolumn{2}{|r|}{42.2} & 20 & 88.7 \\
\hline Weinmannia rollottii (A) & $18 / 27$ & 22.2 & & 51.8 & 12.5 & 86.6 \\
\hline Clusia multiflora (A) & $7 / 27$ & 2 & & 78.1 & 4.2 & 84.2 \\
\hline Brunellia comocladifolia (A) & $16 / 27$ & 47.2 & & 12.2 & 17.9 & 77.3 \\
\hline Clusia alata (A) & $15 / 27$ & 7.8 & & 49.5 & 6.7 & 63.9 \\
\hline Gaultheria rigida (Ar) & $4 / 27$ & 5.2 & & 46.7 & 5.9 & 57.8 \\
\hline Miconia ligustrina $(\mathrm{Ar})$ & $7 / 27$ & 8.7 & & 34.4 & 12.5 & 55.7 \\
\hline & Páram & mo (Arbustal) & & & & \\
\hline Especie & Presencia & CoR & DeR & IPF & FrR & IVI \\
\hline Ageratina tinifolia (Ar) & $3 / 4$ & 24.9 & 9.0 & 56.1 & 13.6 & 44.8 \\
\hline Macleania rupestris (Ar) & $2 / 4$ & 16.1 & 11.1 & 33.9 & 9.1 & 26.9 \\
\hline Symplocos cundinamarcensis (Ar) & $1 / 4$ & 20.3 & 05 & 30.7 & 4.5 & 25.4 \\
\hline Pentacalia tolimensis (Ar) & $1 / 4$ & 11.1 & 4.1 & 30.1 & 4.5 & 23.5 \\
\hline Hypericum lycopodioides (Ar) & $2 / 4$ & 12.3 & 10.6 & 28.1 & 9.1 & 24.9 \\
\hline & Páramc & 10 (Frailejonal) & & & & \\
\hline Especie & Presencia & CoR & & Der & FrR & IVR \\
\hline Espeletia murilloi $(\mathrm{R})$ & $6 / 8$ & 21.5 & & 16.2 & 6.5 & 44.3 \\
\hline Puya goudotiana (R) & $4 / 8$ & 17.5 & & 5.2 & 4.3 & 27.1 \\
\hline Chusquea tessellata $(\mathrm{H})$ & $7 / 8$ & 5.4 & & 8.8 & 7.6 & 21.8 \\
\hline Blechnum auratum $(\mathrm{R})$ & $4 / 8$ & 7.6 & & 2.1 & 4.3 & 14.1 \\
\hline Paepalanthus columbiensis (R) & $4 / 8$ & 1.0 & & 8.5 & 4.3 & 13.9 \\
\hline & Páran & mo (Pajonal) & & & & \\
\hline Especie & Presencia & CoR & & DeR & FrR & IVR \\
\hline Calamagrostis effusa (Gr) & $1 / 1$ & 12.0 & & 21.4 & 15.6 & 49.1 \\
\hline Disterigma empetrifolium $(\mathrm{H})$ & $1 / 1$ & 22.6 & & 8.5 & 9.4 & 40.5 \\
\hline Rhynchospora ruiziana $(\mathrm{H})$ & $1 / 1$ & 7.3 & & 15.9 & 15.6 & 38.8 \\
\hline Neurolepis acuminatissima $(\mathrm{H})$ & $1 / 1$ & 5.9 & & 15.9 & 15.6 & 37.4 \\
\hline Paepalanthus pilosus $(\mathrm{R})$ & $1 / 1$ & 2.7 & & 13.7 & 9.4 & 25.7 \\
\hline
\end{tabular}

A. Árbol; Ar. Arbusto; R. Roseta; H. Hierba; Gr. Gramínea; AbR. Área basal relativa; CoR. Cobertura relativa; DeR. Densidad relativa; DoR. Dominancia relativa; FrR. Frecuencia relativa.

A. Tree; Ar. Bush; R. Roseta; H. Grass; Gr. Gramínea; AbR. Relative basal area; CoR. Relative coverage; DeR. Relative density; DoR. Relative dominance; FrR. Relative frequency. 
estructural que determina la dominancia en los estratos superiores; especies representativas por valores de cobertura son: $C$. multiflora, C. alata, B. comocladifolia, M. rupestris, E. myrtilloides, otras como M. cundinamarcensis, Hesperomeles obtusifolia (Pers.) Lindl., M. funckii, C. caracasana y T. grossa.

Índice de valor de importancia (IVI) en bosque altoandino: taxones con mayor importancia ecológica en Bijagual son: C. elliptica, V. triphyllum, W. rollottii, C. multiflora y $B$. comocladifolia; por valores de densidad relativa: $B$. comocladifolia. Por dominancia relativa: C. elliptica, C. multiflora, W. rollottii, C. alata, G. rigida y D. granadensis. Especies frecuentes son: $V$. triphyllum, B. comocladifolia y $W$. rollottii (Tabla 2).

IPF-IVI en arbustal: en esta comunidad, A. tinifolia, $M$. rupestris y $S$. cundinamarcensis son dominantes (IPF) debido al área basal y cobertura, a su vez, M. rupestris e H. lycopodioides son abundantes. Estas especies presentaron la mayor representatividad ecológica (IVI) por ser frecuentes (Tabla 2).

IVR: en frailejonal fueron E. murilloi, $P$. goudotiana y $C$. tessellata, mientras en pajonal $C$. effusa, D. empetrifolium y $R$. ruiziana (Tabla 2).

\section{DISCUSIÓN}

Diversidad: la probabilidad de diversidad ( $>3.3$ y 2.9 ) y de dominancia $(<0.05$ y 0.03$)$ para bosque altoandino y páramo, se asocian con abundancias proporcionales entre especies; Bijagual es diverso por la riqueza registrada (429 spp.) (Cleef, 1981; Rangel, 1995).

En bosque, tres unidades de muestreo se consideraron dominantes por la presencia de Chusquea; estos transectos están asociados a matrices de pastizal, cultivos de papa y entresaca constante, actividades que ejercen presión sobre la composición y el recambio de individuos; éstas presiones sobre los ecosistemas de alta montaña, también fueron descritos por
Velasco-Linares y Vargas (2008) y, Sarmiento y Llambí (2011). En páramo, la formación de tipo pajonal es homogénea, debido a la dominancia de $C$. effusa, N. acuminatissima y $R$. ruiziana, ya que son abundantes o de amplia cobertura (Tabla 1).

Los transectos con mayor similitud en bosque (16 y 25/46\%) se distancian $6 \mathrm{~km}$ entre sí, y aquellos disimiles (7 y 8/13\%; 12 y $14 / 16 \%$; Fig. 1A) entre 0.3 y $1.6 \mathrm{~km}$; en páramo hay mayor similitud entre frailejonal y arbustal (10-38 \%; Fig. 1B); la baja similitud determina recambio de especies entre unidades de muestreo y posible distribución aleatoria. Este resultado determina diferentes comunidades vegetales dentro de cada ecosistema, definidos por gradientes ambientales (topográficos, climáticos, altitudinales y edáficos), factores biológicos (expresión del banco de semillas germinable y agentes dispersores con capacidad de desplazamiento diferente) (Arroyo-Rodríguez et al., 2015; Lequerica, Bernal, \& Stevenson, 2017), así como transformación espacial y temporal de coberturas vegetales (Gil, 2016), que influyen en las diferentes asociaciones.

Estructura vertical: las formas de crecimiento determinan diferencias fisionómicas para cada ecosistema; en bosque, aunque los árboles (17 \% riqueza; $20 \%$ abundancia) y los arbustos $(15 \% ; 16 \%)$ son elementos conspicuos, éstos para Bijagual no son los más representativos; en su lugar, las hierbas (con alturas hasta de $1.8 \mathrm{~m}$ ), son más abundantes y diversas $(26 \% ; 24 \%)$; estos resultados son similares a los registrados por Alvear, Betancur y Franco-Roselli (2010), Abud y Torres (2016) y Rojas (2017) para bosques altoandinos en Colombia, preservados y en regeneración; así, la estructura y diversidad no solo se define por especies leñosas, la inclusión de todas las formas de crecimiento, en estudios de vegetación, permiten describir el estado de conservación y la funcionalidad asociada a la dinámica natural de las comunidades del bosque (Córdova-Tapia \& Zambrano, 2015). 
Es exuberante la flora epífita ( $25 \%$ riqueza; $19 \%$ abundancia), considerada la segunda forma más diversa del bosque. Estos datos concuerdan con lo registrado por David-Higuita y Álvarez-Dávila (2018), cuya riqueza florística se incrementa por la presencia de herbáceas, terrestres, epífitas y bejucos. Las familias epífitas sobresalientes son: Orchidaceae y Bromeliaceae, así como Pteridophyta, que, en conjunto con la brioflora de Bijagual (IAvH, 2015; Gil \& Morales, 2017), son un componente ecológico importante en suelo y sobre la biomasa ya que constantemente almacenan la humedad, facilitan la disponibilidad de nutrientes, procesos de colonización y hospedaje (Wolf, 2003; RojasFlórez \& Sánchez-Montaño, 2015).

En páramo, el pajonal, arbustal y frailejonal, son estructuralmente similares a otros complejos en Colombia (Morales et al., 2007; Llambí \& Cuesta, 2014); las formas en roseta, macolla y arbusto, no son los más abundantes; sin embargo, en conjunto con las hierbas (mejor representada en abundancia y riqueza), conforman un patrón de distribución vertical típico de zonas de alta montaña, que debido a los procesos de colonización y competencia por espacio, generan heterogeneidad en composición, riqueza y endemismo (Alzate, Jiménez-Montoya, \& Sarrazola, 2016; Jiménez-Rivillas, García, Quijano-Abril, Daza, \& Morrone, 2018). Olaya-Angarita, Díaz-Pérez y Morales-Puentes (2019), en el complejo Guantiva-La Rusia (Boyacá), encuentran comunidades de arbustal con riquezas entre 35-68 spp. y herbazal entre 27-39 spp. (incluido frailejonal); estos datos son similares para los arbustales de Bijagual (66) aunque menores a los frailejonales (73).

Para el bosque altoandino y el páramo, se observó una disminución progresiva en el número de individuos con el aumento en los valores de altura, cobertura y diámetro de tronco (mayor número de individuos en clases inferiores); estos datos concuerdan con los registrado por Samper y Vallejo (2007) y Ávila, Ángel y López (2010), esta organización vertical se asocia con vegetación poco intervenida, con favorable regeneración natural y que experimenta recambio de individuos y proceso de sucesión.

Estructura horizontal: la importancia ecológica de árboles y arbustos, está influenciada por la presencia de individuos de gran porte, principalmente de C. multiflora, C. elliptica, C. alata, W. rollottii, B. comocladifolia, V. triphyllum y E. myrtilloides; estas especies, aunque con valores bajos de densidad, logran los valores más altos de área basal $(>40 \mathrm{~cm})$ y coberturas amplias $\left(46\right.$ y $\left.240 \mathrm{~m}^{2}\right)$.

Lo anterior concuerda con la estructura registrada por Marín y Betancur (1997), Galindo, Betancur y Cadena (2003) y Alvear et al. (2010), para especies de Weinmannia, Clusia, Brunellia y Hedyosmum, consideradas dominantes y con alta importancia ecológica en bosque y páramo. Aunque a nivel de géneros es similar dicha representatividad ecológica, existe variabilidad en la dominancia a nivel de especie, si se comparan estos resultados con otras localidades muestreadas (Galindo et al., 2003; Carvajal, Ariza, Caro, \& Valero, 2014; Carrillo et al., 2017); de esta forma, cada localidad muestra singularidades definidas por la composición y la dinámica estructural. Por lo anterior, este estudio define a Bijagual como un corredor con potencial y alta diversidad biológica.

Finalmente, en el gradiente de elevación (2682-3460 m) se identificaron especies exclusivas de bosque y de páramo; sin embargo, entre los 2900-3000 m (-3200 m), se dan cambios fisionómicos del bosque; especies con porte alto $(>8 \mathrm{~m})$ en bosque, también fueron halladas con porte bajo en el arbustal $(<2 \mathrm{~m})$, dentro de estas C. elliptica, H. colombianum, $W$. rollottii, $W$. fagaroides, $D$. granadensis, $M$. rupestris y $M$. stipularis. Estos resultados son contrapuestos a Olaya-Angarita et al. (2019), con la identificación de cambios fisionómicos entre 3300 y $3649 \mathrm{~m}$ en otros páramos de Boyacá; lo anterior, determina que los cambios fisionómicos de la vegetación en un gradiente de elevación, no son uniformes y que están influenciados por la topografía, la composición de especies, los procesos de dispersión y la 
variación climática (Ramírez, Llambí, Schwarzkopf, Gámez, \& Márquez, 2009; Arzac, Chacón-Moreno, Llambí, \& Dulhoste, 2011).

Declaración de ética: los autores declaran que todos están de acuerdo con esta publicación y que han hecho aportes que justifican su autoría; que no hay conflicto de interés de ningún tipo; y que han cumplido con todos los requisitos y procedimientos éticos y legales pertinentes. Todas las fuentes de financiamiento se detallan plena y claramente en la sección de agradecimientos. El respectivo documento legal firmado se encuentra en los archivos de la revista.

\section{AGRADECIMIENTOS}

A la Universidad Pedagógica y Tecnológica de Colombia, al Herbario UPTC. Al Convenio de cooperación DHS 5211071 Ecopetrol S.A.-UPTC. Al Grupo Sistemática Biológica por el trabajo arduo de campo y laboratorio. A los Herbarios UPTC, HECASA y COL. A O.J. Daza-Leguizamón, C.N. Díaz-Pérez, J.E. Gil-Novoa, M.Y. Carrillo-Fajardo. A, D. Torres del Herbario UPTC. A los especialistas, D.A. Giraldo-Cañas, L.R. Sánchez y M.A. Quijano.

\section{RESUMEN}

Introducción: Los trabajos en Colombia se han acrecentado ante la necesidad de buscar vías para conocer la diversidad que aloja este complejo país y sus ecosistemas, así como la prioridad de protección a lo largo y extenso del mismo. Así, conocer los ecosistemas boyacenses y altoandinos no es la excepción. Objetivo: proporcionar información sobre la diversidad y estructura del Macizo Bijagual, área de alta montaña definida como corredor biológico, en cuyo interior se encuentran formaciones de páramo (2990-3460 m) y bosque altoandino (2682-3268 m). Métodos: El muestreo consistió en 27 transectos aleatorios en bosque altoadino $(100 \times 2.5 \mathrm{~m})$ y 13 en páramo $(10 \times 5 \mathrm{~m})$, se registraron datos de abundancia, forma de crecimiento, altura, cobertura, DAP y área basal. Se realizaron análisis de diversidad (H', D y Jaccard), de estructura vertical y horizontal. Resultados: La riqueza estimada fue de 429 especies en 86 familias, la similitud no supera el $46 \%$ entre unidades de muestreo, dato que apoya la heterogeneidad de Bijagual. Se registraron 10777 individuos en bosque, las hierbas (2595/104) y árboles
(2189/67) fueron los más abundantes y ricos; en páramo 3337 individuos, con dominancia de hierbas (en frailejonal, arbustal y pajonal), rosetas (en frailejonal y pajonal) y arbustos (en arbustal). Especies con representatividad ecológica (IVI) fueron: Clusia multiflora, C. elliptica, C. alata, Weinmannia rollottii, Brunellia comocladifolia y Viburnum triphyllum. Conclusión: Estos resultados confirman la singularidad de estos ecosistemas de alta montaña y la necesidad de mantener la heterogeneidad aun existente ante la incidencia de los procesos de transformación del suelo. Lo anterior, apoyado en el alto recambio de especies entre unidades de muestreo, la dominancia por tipos fisionómicos y la importancia ecológica que exhiben las especies arbóreas y arbustivas en bosque y aquellas frecuentes en páramo. Este estudio define a Bijagual como un corredor con alta diversidad biológica.

Palabras clave: bosque altoandino; páramo; diversidad; formas de crecimiento; vegetación.

\section{REFERENCIAS}

Abud, M., \& Torres, A.M. (2016). Caracterización florística de un bosque alto andino en el Parque Nacional Natural Puracé, Cauca, Colombia. Boletín Científico del Museo de Historia Natural, 20(1), 27-39.

Álvarez-Lopeztello, J., Rivas-Manzano, I.V., AguileraGómez, L.I., \& González-Ledesma, M. (2016). Diversidad y estructura de un pastizal en El Cerrillo, Piedras Blancas, Estado de México, México. Revista Mexicana de Biodiversidad, 87, 980-989.

Alvear, M., Betancur, J., \& Franco-Roselli, P. (2010). Diversidad florística y estructura de remanentes de bosque andino en la zona de amortiguación del Parque Nacional Natural los Nevados, cordillera Central Colombiana. Caldasia, 32(1), 39-63.

Alzate, F., Jiménez-Montoya, J., \& Sarrazola, H. (2016) Sonsón, un nuevo complejo de páramos en Colombia. En M.A. Quijano-Abril (Ed.), Flora del Oriente Antioqueño. Biodiversidad, ecología y estrategias de conservación (pp. 19-24). Bogotá D.C., Colombia: Fondo Editorial Universidad Católica de Oriente.

Armenteras, D., Retana, J., Molowny, R., Roman, R., Gonzalez, F., \& Morales, M. (2011) Characterizing fire spatial pattern interactions with climate and vegetation in Colombia. Agricultural and Forest Meteorology, 151(3), 279-289.

Armenteras, D., Cabrera, E., Rodríguez, N., \& Retana, J. (2013). National and regional determinants of tropical deforestation in Colombia. Regional. Environmental Change, 13(6), 1181-1193.

Arroyo-Rodríguez, V., Melo, F.P.L., Martínez-Ramos, M., Bongers, F., Chazdon, R.L., Meave, J.A., Norden, N., Santos, B.A., Leal, I.R., \& Tabarelli, M. (2015). Multiple successional pathways in human-modified 
tropical landscapes: new insights from forest succession, forest fragmentation and landscape ecology research. Biological Reviews Cambridge Philosophical Society, 92(1), 326-340.

Arzac, A., Chacón-Moreno, E., Llambí, L.D., \& Dulhoste, R. (2011). Distribución de formas de vida de plantas en el límite superior del ecotono bosque páramo en los Andes Tropicales. Ecotrópicos, 24(1), 26-46.

Ávila, F., Ángel, S., \& López, R. (2010). Diversidad y estructura de un robledal en la Reserva Biológica Cachalú, Encino (Santander-Colombia). Revista Colombia Forestal, 13(1), 87-116.

Carrillo, M.Y., Morales-Puentes, M.E., \& Gil-Leguizamón, P.A. (2017). Catálogo de la flora (angiospermas) del páramo de Bijagual, Boyacá, Colombia. Boletín Científico del Centro de Museos, 21(2), 15-29.

Carvajal, R.L.,Ariza, C.W., Caro, P.L., \& Valero, F.N.(2014) Especies forestales representativas del suroriente de Boyacá. Árboles de CORPOCHIVOR-Corporación Autónoma Regional de Chivor-CORPOCHIVOR. Bogotá D.C., Colombia: Universidad Distrital Francisco José de Caldas.

Cleef, A.M. (1981). The vegetation of the paramos of the Colombian dordillera Oriental. Berlín, Alemania: Dissertationes Botanicae.

Cleef, A.M. (2013). Origen, evolución, estructura y diversidad biológica de la alta montaña colombiana. In J. Cortés-Duque \& C. Sarmiento (Eds.), Visión socioeconómica de los páramos y la alta montaña colombiana: memorias del proceso de definición de criterios para la delimitación de páramos (pp. 3-21). Bogotá D.C., Colombia: Instituto de Investigación de Recursos Biológicos Alexander von Humboldt.

Córdova-Tapia, F., \& Zambrano, L. (2015). Functional diversity in community ecology. Ecosistemas, 24(3), 78-87.

David-Higuita, H., \& Álvarez-Dávila, E. (2018). Riqueza total de especies de plantas vasculares en un bosque andino de la cordillera Central de Colombia. Revista de Biología Tropical, 66(1), 227-236.

Etter, A., \& Van Wynngaarden, W. (2000). Patterns of landscape transformation in Colombia, with emphasis in the Andean region. Ambio, 29(7), 432-439.

Font, Q.P. (2001). Diccionario de botánica (2nd Ed.). Barcelona, España: Ediciones Península.

Galindo, R., Betancur, J., \& Cadena, J. (2003). Estructura y composición florística de cuatro bosques andinos del Santuario de Flora y Fauna Guanentá-alto río Fonce, cordillera Oriental colombiana. Caldasia, 25(2), 313-335.

Gentry, A. (1988). Patterns of plant community diversity on geographical and environmental gradients. Annals of the Missouri Botanical Garden, 75(1), 1-52.
Gentry, A. (1993). A field guide to the families and genera of woody plants of northwest South America (Colombia, Ecuador, and Peru) with supplementary notes on herbaceous taxa. Washington, USA: Conservation International.

Gil, P.A., \& Morales, M.E. (2015). Multitemporalidad de coberturas vegetales del complejo páramo de Bijagual (Boyacá). Memorias VIII Congreso Colombiano de Botánica.

Gil, P.A. (2016). Análisis multitemporal de la vegetación del Macizo de Bijagual, Boyacá. (Tesis de Maestría). Universidad Pedagógica y Tecnológica de Colombia.

Gil, J.E., \& Morales, M.E. (2017). Briófitos del complejo de páramos Tota-Bijagual-Mamapacha: un acercamiento a su riqueza y fitogeografía. Ciencia en Desarrollo, $1 E$ (Suplemento Especial), 156-157.

Hammer, O., Harper, D.A.T., \& Ryan, P.D. (2008). PASTPalaeontological Statistic, ver. 1.77. Retrieved from http://www.folk.unio.no/ohammer/past

IAvH. (2015). Caracterización biótica del complejo de páramos Tota-Bijagual-Mamapacha en jurisdicción de Corpoboyacá. Convenio No. 14-13-014-195CE. Bogotá, Colombia: Instituto de Investigaciones de Recursos Biológicos Alexander von Humboldt.

Jiménez-Rivillas, C., García, J.J., Quijano-Abril, M.A., Daza, J.M., \& Morrone, J.J. (2018). A new biogeographical regionalisation of the Páramo biogeographic province. Australian Systematic Botany, 31, 296-310.

Lequerica, M., Bernal, M., \& Stevenson, P. (2017). Evidencia de direccionalidad del proceso de sucesión temprana del bosque altoandino. Colombia Forestal, 20(1), 63-84.

Llambí, L.D., \& Cuesta, F. (2014). La diversidad de los páramos andinos en el espacio y en el tiempo. En F.J. Cuesta, L. Sevink, B. Llambí, \& J. Posner (Eds.), Avances en investigación para la conservación de los páramos andinos. Quito, Ecuador: CONDESAN.

Marín, C., \& Betancur, J. (1997). Estudio florístico en un robledal del Santuario de Flora y Fauna de Iguaque (Boyacá-Colombia). Academia Colombiana de Ciencias Exactas, Físicas y Naturales, 21(80), 249-259.

Morales, M., Otero, J., van der Hammen, T., Torres, A., Cadena, C., Pedraza, C., ... Cárdenas, L. (2007). Atlas de páramos de Colombia. Bogotá D.C., Colombia: Instituto de Investigación de Recursos Biológicos Alexander von Humboldt.

Morales-Puentes, M.E., Gil-Leguizamón, P.A., Simbaqueba-Gutiérrez, A.L., \& Carrillo-Fajardo, M.Y. (2012). Biodiversidad y objetos de conservación de los ecosistemas estratégicos de Sucuncuca y Bijagual (Provincia de Lengupá) Boyacá (Informe final convenio 
de colaboración DHS N5211071). Tunja, Colombia: Universidad Pedagógica y Tecnológica de Colombia.

Olaya-Angarita, J.A., Díaz-Pérez, C.N., \& Morales-Puentes, M.E. (2019). Composición y estructura de la transición bosque-páramo en el corredor GuantivaLa Rusia (Colombia). Revista de Biología Tropical, 67(4), 755-768.

Ramírez, L., Llambí, L.D., Schwarzkopf, T., Gámez, L.E., \& Márquez, N.J. (2009). Vegetation structure along the forest-paramo transition belt in the Sierra Nevada de Mérida: Implications for understanding treeline dynamics. Ecotropicos, 22(2), 83-98.

Rangel, J.O. (1995). Consideraciones sobre la diversidad vegetal de alta montaña en Colombia. En J.A. Lozano \& J.B. Pabón (Eds.), Memorias del seminario taller sobre alta montaña colombiana (pp. 33-60). Bogotá, Colombia: Editora Guadalupe.

Rangel, J.O., \& Velásquez, A. (1997). Métodos de estudio de la vegetación. En J.O. Rangel, C. Lowy, \& M. Aguilar (Eds.), Colombia Diversidad Biótica II: tipos de vegetación en Colombia (pp. 59-87). Bogotá D.C., Colombia: Editorial Unibiblos.

Rojas, S.L. (2017). Estructura y composición florística de la vegetación en proceso de restauración en los Cerros Orientales de Bogotá (Colombia). Caldasia, 39(1), 124-139.
Rojas-Flórez., C.B., \& Sánchez-Montaño, L.R. (2015). Estructura espacial de epífitas vasculares en dos localidades de bosque altoandino, Pamplona, Colombia. Caldasia, 37(1), 15-30.

Samper, C., \& Vallejo, M. (2007). Estructura y dinámica de poblaciones de plantas en un bosque andino. Revista de la Academia Colombiana de Ciencias Exactas, Físicas y Naturales, 31(118), 57-68.

Sarmiento, L., \& Llambí, L.D. (2011). Regeneración del páramo luego de un disturbio agrícola: una síntesis de 20 años de investigaciones en sistemas con descansos largos en la cordillera de Mérida. En F. Herrera, \& I. Herrera (Eds.), La restauración ecológica en Venezuela: fundamentos y experiencias (pp. 123-148). Caracas, Venezuela: Ediciones Instituto Venezolano de Investigaciones Científicas.

Velasco-Linares, P., \& Vargas, O. (2008). Problemática de los bosques alto andinos. En O. Vargas (Ed.), Estrategias para la restauración ecológica del bosque alto andino (El caso de la Reserva Forestal Municipal de Cogua, Cundinamarca) (pp. 41-56). Bogotá, Colombia: Universidad Nacional de Colombia.

Wolf, J. (2003). Diversidad y ecología de comunidades epífitas en la cordillera Central, Colombia. En T. Van der Hammen \& A. Dos Santos (Eds.), Estudios de ecosistemas tropandinos (pp. 453-502). Bogotá D.C., Colombia: Instituto Geográfico "Agustín Codazzi" (IGAC). 